\title{
Effect of Chronic Obstructive Pulmonary Disease on Swallowing Function in Stroke Patients
}

\author{
Gun Woong Park, MD' ${ }^{1}$, Suk Kyoung Kim, MD ${ }^{1}$, Chang Hwa Lee, MD¹, \\ Chung Reen Kim, $\mathrm{MD}^{1}$, Ho Joong Jeong, $\mathrm{MD}^{2}$, Dong Kyu Kim, $\mathrm{MD}^{2}$ \\ ${ }^{1}$ Department of Rehabilitation Medicine, Dong-Eui Medical Center, Busan; \\ ${ }^{2}$ Department of Physical Medicine and Rehabilitation, Kosin University College of Medicine, Busan, Korea
}

Objective To investigate the prevalence of chronic obstructive pulmonary disease (COPD) in stroke patients, and to assess the difference in swallowing function between stroke patients with COPD (COPD group) and stroke patients without COPD (control group).

Methods The subjects included 103 stroke patients. They underwent the pulmonary function test and were assigned to either the COPD group or the control group. Their penetration-aspiration scale (PAS) scores and functional dysphagia scale scores were compared by performing a videofluoroscopic swallowing study. The intergroup differences in lip closure, bolus formation, mastication, and the oral transit time, laryngeal elevation, cricopharyngeal dysfunction, oronasal regurgitation, residue in pyriform sinus and vallecula, pharyngeal transit time, aspiration, and esophageal relaxation were also compared.

Results Thirty patients were diagnosed with COPD. The COPD group showed statistically higher PAS scores (4.67 \pm 2.15$)$ compared to the control group $(2.89 \pm 1.71)$. Moreover, aspiration occurred more frequently in the COPD group with statistical significance $(\mathrm{p}<0.05)$. The COPD group also showed higher occurrence of cricopharyngeal dysfunction, albeit without statistical significance.

Conclusion This study shows that a considerable number of stroke patients had COPD, and stroke patients with COPD had higher risk of aspiration than stroke patients without COPD.

Keywords Stroke, Chronic obstructive pulmonary disease (COPD), Deglutition disorders, Respiratory aspiration

Received August 7, 2014; Accepted September 26, 2014

Corresponding author: Dong Kyu Kim

Department of Physical Medicine and Rehabilitation, Kosin University College of Medicine, 262 Gamcheon-ro, Seo-gu, Busan 602-702, Korea

Tel: +82-51-990-6361, Fax: +82-51-241-2019, E-mail: peyous@daum.net

(c) This is an open-access article distributed under the terms of the Creative Commons Attribution Non-Commercial License (http://creativecommons. org/licenses/by-nc/3.0) which permits unrestricted noncommercial use, distribution, and reproduction in any medium, provided the original work is properly cited.

Copyright $\odot 2015$ by Korean Academy of Rehabilitation Medicine

\section{INTRODUCTION}

Dysphagia affects $37 \%$ to $78 \%$ of patients with acute stroke and is associated with increased risk of aspiration, pneumonia, disability, and death [1]. Dysphagia, one of the common complications of stroke, is also known to occur in chronic obstructive pulmonary disease (COPD). COPD is known to be caused by smoking, repeated airway infections, air pollution, exposure to dust in the workplace, and genetic factors [2], among which smoking 
is the single most important risk factor [3].

Furthermore, smoking is also a risk factor for cardiovascular disease, and it is known to cause cerebral infarction and cerebral and subarachnoid hemorrhage [4-6]. In addition to smoking, aging is one of the risk factors for COPD [7], and stroke is also more common in the elderly. Therefore, the risk of stroke in COPD patients is estimated to be high, and although attention needs to be paid to the issues arising when these diseases accompany each other, to date, there has been no study on this issue.

There are few studies on dysphagia in COPD patients. In the study by Good-Fratturelli et al. [8] on veterans, dysphagia was found in approximately $85 \%$ of COPD patients, and penetration or aspiration was observed in $70 \%$ of COPD patients upon a videofluoroscopic swallowing study (VFSS). In addition, in the study by Cvejic et al. [9], aspiration was observed in about $60 \%$ of COPD patients, suggesting that aspiration is caused by a lack of interaction between breathing and swallowing [10]. In addition to aspiration, since COPD is reportedly accompanied by cricopharyngeal dysfunction [11], the COPD status in stroke patients is estimated to have a considerable influence on dysphagia. Stroke and COPD are different causes of dysphagia. We thought that if stroke and COPD coexist, a more severe dysphagia is present.

Therefore, in this study, we aimed to examine the prevalence of COPD in stroke patients and to determine the effects on the swallowing function in stroke patients with COPD through a VFSS.

\section{MATERIALS AND METHODS}

\section{Subjects}

The subjects included this study were stroke patients within 3 months of stroke onset. Patients who were older than 60 years; who were hospitalized and treated in Dong-Eui Medical Center from January 2014 to June 2014; and from whom informed consent for participating in this study could be obtained, either from the patients themselves or from their families, were included in this study. Patients who had no history of previous stroke were selected as the study subjects, and cerebral infarction and cerebral hemorrhage were confirmed by brain magnetic resonance images and brain computed tomography scans. Patients who had previously experienced a stroke were being treated for complications such as pneumonia, were unmovable because they were admitted to the intensive care unit, had cognitive function impairment, as determined by a Korean version of Mini-Mental State Examination score below 21, or were intubated with a tracheostomy tube were excluded from our analysis. Institutional Review Board of the Dong-Eui Medical Center approved the study protocol (No. DEMC2014-01).

\section{Methods}

This study was conducted progressively as a patientcontrol group study. Through pulmonary function tests, the subjects were divided into the COPD group and the normal (control) group, and the baseline differences between the two groups were compared. Subsequently, dysphagia was evaluated by a VFSS.

An internal medicine specialist and a rehabilitation medicine specialist participated in the pulmonary function testing, which was performed by a clinical pathologist. Forced vital capacity (FVC), forced expiratory volume in the first second (FEV1), and the FEV1/FVC ratio (the ratio of FEV1 to FVC) were measured; patients with an FEV1/ FVC ratio below $70 \%$ were classified as having COPD [12].

VFSS was performed according to a modified version of Logemann's protocol [13]. A rehabilitation medicine specialist directly performed the test in the fluoroscopy room, and the subjects were seated and asked to swallow diluted barium during fluoroscopy. Barium was diluted in water (barium/water, 35/65\% weight/volume ratio),

Table 1. The penetration-aspiration scale

\begin{tabular}{cl}
\hline Score & \multicolumn{1}{c}{ Description } \\
\hline 1 & Contrast does not enter the airway \\
2 & $\begin{array}{c}\text { Contrast enter the airway, remains above vocal } \\
\text { folds; no residue }\end{array}$ \\
\hline 3 & $\begin{array}{c}\text { Contrast remains above vocal folds, visible } \\
\text { residue remains }\end{array}$ \\
& Contrast contacts vocal folds, no residue \\
\hline 5 & $\begin{array}{c}\text { Contrast contacts vocal folds, visible residue } \\
\text { remains }\end{array}$ \\
& Contrast passes glottis, no subglottic residue \\
& visible \\
7 & Contrast passes glottis, visible subglottic \\
& residue despite patient's response \\
\hline 8 & Contrast passes glottis, visible subglottic \\
& residue, absent patient response \\
\hline
\end{tabular}


and the tests were conducted using a volume of $20 \mathrm{~mL}$. VFSS was performed considering the dysphagia level, general condition, and cooperation level of each patient. The pulmonary function test and VFSS were performed on the same day.

VFSS was performed, and two rehabilitation medicine specialists evaluated the video recordings. The levels of penetration and aspiration on VFSS were observed and scored based on the penetration-aspiration scale (PAS) designed by Rosenbek et al. [14] (Table 1) for objectivity. It was considered that the higher the score, the higher the aspiration risk. VFSS was used to evaluate the functional dysphagia scale (FDS) [15]. In addition, lip closure, bolus formation, mastication, and the oral transit time were measured in the oral phase. Normal oral transit time was defined as less than 1 second. In the pharyngeal phase, laryngeal elevation, cricopharyngeal dysfunction, oronasal regurgitation, residues in the valleculum and pyriform sinus, the pharyngeal transit time, and the aspiration status were observed. Residues in the valleculum and pyriform sinus less than $10 \%$ were considered normal, and the normal pharyngeal transit time was defined as less than 1 second. Aspiration was defined when the bolus passed through the vocal folds. Furthermore, in the esophageal phase, the esophageal relaxation status was examined.

Statistical analysis was performed with SPSS ver. 18.0 (SPSS Inc., Chicago, IL, USA) for Windows statistical software. The correlation between COPD and the risk of aspiration in the COPD and control groups was comparatively analyzed by the Mann-Whitney U test based on the PAS and FDS. The correlations of dysphagia differences in the oral, pharyngeal, and esophageal phases were analyzed using the Pearson chi-square test. The results are presented as mean \pm standard deviation or percent. A pvalue of $<0.05$ was considered statistically significant.

\section{RESULTS}

\section{Demographic characteristics of the study subjects}

A total of 120 stroke patients were enrolled in our study. However, accurate tests could not be performed in 17 patients because of low levels of cooperation during the pulmonary function tests and VFSS, and hence these patients were excluded from the study. Among the remaining 103 patients, 53 were males and 50 were females; there were 61 patients with cerebral ischemic infarction and 42 patients with cerebral hemorrhage.

Based on the result of the pulmonary function tests, the number of patients diagnosed with COPD, as determined by an FEV1/FVC ratio below $70 \%$, was 30 (29.1\%) and that of control patients was $73(70.9 \%)$. The test was performed on average $34.9 \pm 14.3$ days after stroke in the COPD group and on average $36.5 \pm 18.3$ days after stroke in the control group; and the mean ages of patients in the COPD and control groups were $73.4 \pm 6.3$ years and $71.9 \pm 5.5$ years, respectively. There were no significant differences between the two groups ( $p>0.05$ ). With respect to the affected region, 15,10 , and 5 patients in the COPD group developed cortical, subcortical, and brainstem lesions; and 43, 19, and 11 patients in the control group developed cortical, subcortical, and brainstem lesions, respectively; there were no significant differences between the two groups $(p>0.05)$. In terms of smoking history, 25 patients $(83.3 \%)$ in the COPD group smoked $33.8 \pm 4$ packs/year on average, while 37 patients $(50.6 \%)$ in the control group smoked an average of $33.7 \pm 5.5$ packs/year; the smoking rate in the COPD group was significantly higher than that in the control group (Table 2).

Table 2. Demographic and clinical characteristics of all subjects in the case-control study

\begin{tabular}{lcc}
\hline \multicolumn{1}{c}{ Characteristic } & $\begin{array}{c}\text { COPD } \\
(\mathbf{n = 3 0 )}\end{array}$ & $\begin{array}{c}\text { Control } \\
(\mathbf{n = 7 3 )}\end{array}$ \\
\hline Age (yr) & $73.4 \pm 6.3$ & $71.9 \pm 5.5$ \\
Gender (male:female) & $14: 16$ & $39: 34$ \\
Stroke side (right:left) & $13: 17$ & $32: 41$ \\
Stroke type (ischemic:hemorrhagic) & $18: 12$ & $43: 30$ \\
Location of lesion & & \\
Cortex & $15(50.0)$ & $43(59.0)$ \\
\hline Subcortex & $10(33.0)$ & $19(26.0)$ \\
Brainstem & $5(17.0)$ & $11(15.0)$ \\
Days after stroke & $34.9 \pm 14.3$ & $36.5 \pm 18.3$ \\
Smoking & $25(83.0)^{*}$ & $37(50.0)^{*}$ \\
Smoking amount (pack/yr) & $33.8 \pm 4$ & $33.7 \pm 5.5$ \\
\hline
\end{tabular}

Values are presented as mean \pm standard deviation or number (\%).

COPD, chronic obstructive pulmonary disease. ${ }^{*} \mathrm{p}<0.05$. 
Differences in VFSS between the COPD and control groups

As determined by VFSS, the aspiration risk was significantly higher among stroke patients in the COPD group as compared to stroke patients in the control group based on the PAS score ( $4.67 \pm 2.15$ vs. $2.89 \pm 1.71 ; \mathrm{p}<0.05)$ (Table 3 ).

There were no significant differences in the FDS score between the two groups $(22.30 \pm 10.31$ vs. $20.11 \pm 8.15$; $\mathrm{p}=$ 0.216 ). No statistically significant differences were observed between the two groups in terms of lip closure, bolus formation, status of mastication impairment, and oral transit time during the oral phase. Moreover, in the pharyngeal phase, there were no statistically significant differences between the two groups in terms of laryngeal elevation, cricopharyngeal dysfunction, oronasal regurgitation, residues in the valleculum and pyriform sinus, and pharyngeal transit time. However, there were statistically significant differences in the aspiration status, 12 patients (40.0\%) in the COPD group experienced aspiration, compared to 9 patients (12.3\%) in the control group $(\mathrm{p}=0.002)$. Furthermore, there was no significant difference between the two groups in terms of the esophageal relaxation status in the esophageal phase $(p>0.05)$ (Table 3$)$.

\section{DISCUSSION}

We investigated the COPD prevalence in stroke patients, its association with smoking, and its impact on dysphagia. The COPD prevalence among subjects in this study was $29.1 \%$, and it was found to be highly associated with the high smoking rate in the COPD group. In addition, the aspiration risk in the COPD group was higher than that in the control group, and this was thought to be related with deterioration of the respiratory function causing worsening of dysphagia, especially aspiration risk.

COPD manifests in the form of bronchitis and emphysema, and its clinical symptoms include cough, sputum, and dyspnea on exertion. It also causes arterial wall thickening and endothelial dysfunction, and it is thus a risk factor for myocardial and cerebral infarction. Furthermore, it can cause systemic symptoms such as inflammation, skeletal muscle weakness, and osteoporosis [16]. As the average life span of humans has increased in recent years, the prevalence of COPD has increased simultaneously. According to the Global Initiative for Chronic Obstructive Lung Disease (GOLD) diagnosis

Table 3. Differences in VFSS between the COPD and control groups

\begin{tabular}{|lccc}
\hline & COPD $(\mathbf{n}=\mathbf{3 0})$ & Control $(\mathbf{n}=\mathbf{7 3})$ & p-value \\
\hline Penetration-aspiration scale score & $4.67 \pm 2.15$ & $2.89 \pm 1.71$ & $0.000^{*}$ \\
\hline Functional dysphagia scale score & $22.30 \pm 10.31$ & $20.11 \pm 8.15$ & 0.216 \\
\hline Oral phase & & & \\
\hline Impaired lip closure & $7(23.3)$ & $18(24.6)$ & 0.887 \\
\hline Impaired bolus formation & $9(30.0)$ & $26(35.6)$ & 0.585 \\
\hline Impaired mastication & $9(30.0)$ & $19(26.0)$ & 0.681 \\
\hline Delayed oral transit time & $16(53.3)$ & $28(38.3)$ & 0.180 \\
\hline Pharyngeal phase & & & 0.503 \\
\hline Impaired laryngeal elevation & $19(63.3)$ & $32(43.8)$ & $0.089^{* *}$ \\
\hline Cricopharyngeal dysfunction & $17(56.6)$ & $28(38.3)$ & 0.117 \\
\hline Oronasal regurgitation & $1(3.3)$ & $0(0.0)$ & 0.829 \\
\hline Residue in valleculae & $8(26.6)$ & $21(28.7)$ & 0.548 \\
\hline Residue in pyriform sinus & $13(43.3)$ & $27(36.9)$ & 0.872 \\
\hline Delayed pharyngeal transit time & $19(63.3)$ & $45(61.6)$ & $0.002^{*}$ \\
\hline Aspiration & $12(40.0)$ & $9(12.3)$ & 0.543 \\
\hline Esophageal phase & & & $9(12.3)$ \\
\hline Impaired esophageal relaxation & $5(16.6)$ & & \\
\hline
\end{tabular}

Values are presented as mean \pm standard deviation or number (\%).

VFSS, videofluoroscopic swallowing study; COPD, chronic obstructive pulmonary disease.

${ }^{*} \mathrm{p}<0.05,{ }^{* *} \mathrm{p}<0.1$. 
standard in the United States, $25 \%$ of elderly people aged over 75 years develop COPD [17]. In Korea, the COPD prevalence among people 45 years or older in 2011 was $12.0 \%$, and the corresponding rate was $30.7 \%$ among elderly people aged over 60 years [18]. In this study, because of assessment of stroke patients aged above 60 years, the COPD prevalence was found to be approximately $29.1 \%$, which is similar to the above-mentioned results. Although a higher COPD prevalence was expected in stroke patients, since smoking is known to be the most important risk factor for stroke as well as for COPD, no large differences compared to the prevalence in the normal elderly population were observed. However, we speculate that this may be due to the difficulty in confirming the accurate prevalence as the number of patients in this study was small, and because patients in whom pulmonary function tests could not be performed were excluded from our analysis.

On the other hand, dysphagia is a common complication after stroke, and previous studies have reported that dysphagia occurs in $51 \%-73 \%$ of acute stroke patients $[19,20]$. Various dysphagia symptoms appear secondary to brainstem and cerebral lesions in stroke [21], and these include loss of sensation, impairment of tongue movements, and loss of mastication function in the oral phase, and delayed swallowing reflex, decreased laryngeal elevation or closure of the epiglottis, and cricopharyngeal dysfunction in the pharyngeal phase. It is known that dysphagia is observed in approximately $85 \%$ of COPD patients on VFSS [8]. In this study, the aspiration risk and aspiration status were significantly higher in the COPD group than in the control group. But, FDS score did not show a statistically significant difference between the two groups.

The aspiration risk was especially high in stroke patients with COPD in this study, and this is similar to the results reported in previous studies on general COPD patients. Shaker et al. [10] explained the reason for the high aspiration risk in these patients as follows: COPD has the potential to disrupt the coordination of breathing and swallowing because of tachypnea, an increased tendency to swallow during inspiration, reduced duration of apnea, and changes in the mechanics of swallowing. Patients with COPD tend to swallow during inspiration and swallowing is often followed by inspiratory activity. Inspiration just before or just after a swallow is likely to increase the risk of inhaling pharyngeal contents because of negative intrathoracic pressure [22].

Caution is needed since aspiration during dysphagia may cause pneumonia, hinder rehabilitation treatment, and deteriorate patients' conditions. According to a previous report, aspiration pneumonia is responsible for $34 \%$ of deaths among acute stroke patients, and it is the third most common cause of death secondary to stroke [23]. The probability of pneumonia in stroke patients in whom aspiration is observed on VFSS is reportedly 6-20 times higher than that in those in whom aspiration is not observed on VFSS [23]. In this study, the VFSS showed that stroke patients with COPD had a mean PAS score of $4.67 \pm 2.15$, which was significantly higher than that in stroke patients without COPD. Moreover, the actual aspiration rate in the COPD group was $40.0 \%$, which was significantly higher than that in the control group (12.3\%). This difference was thought to be due to the breathing and swallowing difficulties mentioned above which accompany COPD. Since the aspiration risk is elevated due to dysphagia caused by stroke, the fact that COPD patients start inhaling during swallowing is thought to be the reason why aspiration is more frequently observed in these patients than in stroke patients without COPD.

On the other hand, according to the study by Stein et al. [11], COPD patients have cricopharyngeal dysfunction. In this study, although there was no statistically significant difference in cricopharyngeal dysfunction between the two groups, the proportion of patients with cricopharyngeal dysfunction was higher in the COPD group than in the control group. The cricopharyngeal muscle contracts between the inhalation stage and swallowing, and it relaxes when the food passes through. This prevents regurgitation of food from the stomach or esophagus during the inhalation stage. Thus, dysfunction of the cricopharyngeal muscle causes dysphagia and makes passage of food through the upper esophageal sphincter difficult, often resulting in a small amount of aspiration. More aspiration may ensue as this action is repeated, which in turn may damage the esophageal epithelial cells and lung parenchyma. In previous studies on this topic, dysfunction of the cricopharyngeal muscle was observed in $80 \%$ of COPD patients [11]. However, the cause of cricopharyngeal dysfunction in patients with COPD is not yet known. Belsey [24] suggested that gastroesophageal reflux disease (GERD) leads to cricopharyngeal dysfunc- 
tion, a mechanism that protects the larynx from aspiration of gastric acid. Since it can be speculated that COPD may cause increased gastroesophageal reflux due to flattening of the diaphragm, the presence of COPD may lead to progressive cricopharyngeal dysfunction. In this study, however, it could not be verified whether the patients with COPD developed GERD or not. The VFSS confirms the presence of reflux in only a minority of patients with symptomatic reflux disease [25]; hence, it would be useful to verify the presence of GERD in patients with COPD by performing continuous $\mathrm{pH}$ monitoring to determine the degree of cricopharyngeal dysfunction more accurately.

Because the aspiration risk in stroke patients with COPD was found to be high in this study, we believe that, in general, these patients should be treated not only for dysphagia but also for COPD. A bronchodilator or inhaled steroids can be used to improve the breathing function, and, if necessary, the breathing and swallowing functions can be restored to the normal state by oxygen treatment to lower hypoxemia. As a rehabilitation treatment, increasing muscular strength and endurance of the diaphragm through treatment of swallowing disorders, as well as training of the respiratory muscles and improving the ventilation, can allow smooth oxygen delivery to the tissues, improve the energy and quality of life of the patient, and delay development of fatigue of the respiratory muscles, thereby preventing development of respiratory failure. Although the treatment effects were not examined in this study, these treatments can be expected to reduce dysphagia and aspiration, as well as the incidence of ensuing aspiration pneumonia. Diverse future studies related to this issue might be helpful in determination of the most appropriate treatment.

We believe that the easiest and most effective way to reduce aspiration risk in stroke patients with COPD is by smoking cessation, as a result of which the incidence of COPD can be drastically reduced. Importantly, smoking cessation can also simultaneously reduce the incidence of stroke. As seen in general COPD patients, the COPD group showed a high smoking rate in this study. The smoking rate of $83.3 \%$ in the COPD group was significantly higher than the smoking rate of $50.6 \%$ in the control group. This finding is similar to the results reported in a previous study, in which $70 \%$ of patients diagnosed with COPD were smokers [26]. In addition, smoking promotes atherosclerosis by acting on the cardiovascular system, and it increases the risk of brain infarction by causing platelet aggregation and vascular occlusion. Smoking also causes cerebral and subarachnoid hemorrhage because of rupture of aneurysms or a damaged small intraparenchymal artery [27]. Therefore, lowering the smoking rate is important in preventing both COPD and stroke. According to a previous study, the stroke risk of a smoker decreases to the level of a non-smoker if the smoker stops smoking for 5 years [28]. However, according to another report, although the smoking cessation rate in elderly people, who have a relatively high risk of stroke, is higher than that in young people, doctors generally did not advise elderly patients to stop smoking [29]. Thus, we believe that it is necessary to lower the incidence of COPD and stroke by more active smoking cessation initiatives.

There are a number of limitations to this study. First, since we only selected the subjects having a good general condition and who cooperated well, it was difficult to identify the accurate COPD prevalence among stroke patients because of the limited number of subjects; it was also difficult to compare the severity of COPD or stroke between the groups. Future large-scale studies on the correlation between COPD and stroke can be expected to be helpful for the treatment of dysphagia. Second, although we examined the aspiration risk, we did not assess its correlation with the incidence of actual aspiration pneumonia owing to difficulties in long-term observation. Third, we did not examine the degree of improvement in aspiration and dysphagia with COPD treatment; however, we intend to investigate this aspect in the future. Finally, we did not examine the impact on other functional disorders besides dysphagia in stroke patients with COPD. Since deterioration of the breathing function can influence the general condition of patients, it would be helpful if the impact of COPD on stroke recovery is determined in future studies.

In conclusion, we found that a considerable number of stroke patients had COPD, and that COPD increased the risk of dysphagia, particularly the aspiration risk, in stroke patients. The risk of COPD complications was especially high in aspirating stroke patients; and therefore, we believe that it is necessary to evaluate the status of respiratory disease, such as COPD, through pulmonary function tests in all stroke patients. If proper COPD treatment is performed upon diagnosis, this may aid in the functional recovery after stroke, including improvement 
in the symptoms of dysphagia. However, additional studies are needed on this topic.

\section{CONFLICT OF INTEREST}

No potential conflict of interest relevant to this article was reported.

\section{REFERENCES}

1. Martino R, Foley N, Bhogal S, Diamant N, Speechley M, Teasell R. Dysphagia after stroke: incidence, diagnosis, and pulmonary complications. Stroke 2005;36:2756-63

2. Brannon FJ, Foley MW, Starr JA, Black MG. Cardiopulmonary rehabilitation; basic theory and application. 2nd ed. Philadelphia: F. A. Davis; 1993.

3. Chapman KR, Mannino DM, Soriano JB, Vermeire PA, Buist AS, Thun MJ, et al. Epidemiology and costs of chronic obstructive pulmonary disease. Eur Respir J 2006;27:188-207.

4. Ohira T, Shahar E, Chambless LE, Rosamond WD, Mosley TH Jr, Folsom AR. Risk factors for ischemic stroke subtypes: the Atherosclerosis Risk in Communities study. Stroke 2006;37:2493-8.

5. Kurth T, Kase CS, Berger K, Schaeffner ES, Buring JE, Gaziano JM. Smoking and the risk of hemorrhagic stroke in men. Stroke 2003;34:1151-5.

6. Kurth T, Kase CS, Berger K, Gaziano JM, Cook NR, Buring JE. Smoking and risk of hemorrhagic stroke in women. Stroke 2003;34:2792-5.

7. Kojima S, Sakakibara H, Motani S, Hirose K, Mizuno F, Ito M, et al. Effects of smoking and age on chronic obstructive pulmonary disease in Japan. J Epidemiol 2005;15:113-7.

8. Good-Fratturelli MD, Curlee RF, Holle JL. Prevalence and nature of dysphagia in VA patients with COPD referred for videofluoroscopic swallow examination. J Commun Disord 2000;33:93-110.

9. Cvejic L, Harding R, Churchward T, Turton A, Finlay P, Massey D, et al. Laryngeal penetration and aspiration in individuals with stable COPD. Respirology 2011;16:269-75.

10. Shaker R, Li Q, Ren J, Townsend WF, Dodds WJ, Martin BJ, et al. Coordination of deglutition and phases of respiration: effect of aging, tachypnea, bolus volume, and chronic obstructive pulmonary disease. Am J Physiol 1992;263(5 Pt 1):G750-5.

11. Stein M, Williams AJ, Grossman F, Weinberg AS, Zuckerbraun L. Cricopharyngeal dysfunction in chronic obstructive pulmonary disease. Chest 1990;97:347-52.

12. Reilly J, Silverman EK, Shapiro SD. Chronic obstructive pulmonary disease. In: Longo D, Fauci AS, Kasper D, Hauser SL, Jameson JL, editors. Harrison's principles of internal medicine. 18th ed. New York: McGraw-Hill; 2011. p. 2151-9.

13. Logemann JA. Manual for the videofluorographic study of swallowing. 2nd ed. Austin: Pro-Ed; 1993.

14. Rosenbek JC, Robbins JA, Roecker EB, Coyle JL, Wood JL. A penetration-aspiration scale. Dysphagia 1996; 11:93-8.

15. Han TR, Paik NJ, Park JW. Quantifying swallowing function after stroke: A functional dysphagia scale based on videofluoroscopic studies. Arch Phys Med Rehabil 2001;82:677-82.

16. Yoo CG. Pathogenesis and pathophysiology of COPD. Korean J Med 2009;77:383-400.

17. Mannino DM. COPD: epidemiology, prevalence, morbidity and mortality, and disease heterogeneity. Chest 2002;121(5 Suppl):121S-126S.

18. Kim J, Rhee CK, Yoo KH, Kim YS, Lee SW, Park YB, et al. The health care burden of high grade chronic obstructive pulmonary disease in Korea: analysis of the Korean Health Insurance Review and Assessment Service data. Int J Chron Obstruct Pulmon Dis 2013;8:561-8.

19. Daniels SK, Brailey K, Priestly DH, Herrington LR, Weisberg LA, Foundas AL. Aspiration in patients with acute stroke. Arch Phys Med Rehabil 1998;79:14-9.

20. Gordon C, Hewer RL, Wade DT. Dysphagia in acute stroke. Br Med J (Clin Res Ed) 1987;295:411-4.

21. Heimlich HJ. Rehabilitation of swallowing after stroke. Ann Otol Rhinol Laryngol 1983;92(4 Pt 1):357-9.

22. Gross RD, Atwood CW Jr, Ross SB, Olszewski JW, Eichhorn KA. The coordination of breathing and swallowing in chronic obstructive pulmonary disease. Am J Respir Crit Care Med 2009;179:559-65.

23. Horner J, Massey EW. Silent aspiration following stroke. Neurology 1988;38:317-9.

24. Belsey R. Functional disease of the oesophagus. Postgrad Med J 1963;39:290-8.

25. Groher ME, Crary MA. Dysphagia: clinical manage- 
ment in adults and children. Maryland Heights: Mosby; 2010.

26. Reilly J, Silverman EK, Shapiro SD. Chronic obstructive pulmonary disease. In: Braunwald E, Fauci AS, Kasper D, Hauser SL, Longo DL, Jameson JL, editors. Harrison's principles of internal medicine. 16th ed. New York: McGraw-Hill; 2005. p. 1147-50.

27. Shah RS, Cole JW. Smoking and stroke: the more you smoke the more you stroke. Expert Rev Cardiovasc
Ther 2010;8:917-32.

28. Wolf PA, D'Agostino RB, Kannel WB, Bonita R, Belanger AJ. Cigarette smoking as a risk factor for stroke: the Framingham Study. JAMA 1988;259:1025-9.

29. Maguire CP, Ryan J, Kelly A, O'Neill D, Coakley D, Walsh JB. Do patient age and medical condition influence medical advice to stop smoking? Age Ageing 2000;29:264-6. 